\title{
Video Article \\ Atomic Layer Deposition of Vanadium Dioxide and a Temperature-dependent Optical Model
}

\author{
Marc Currie ${ }^{1}$, Michael A. Mastro ${ }^{1}$, Virginia D. Wheeler ${ }^{1}$ \\ ${ }^{1}$ Naval Research Laboratory \\ Correspondence to: Marc Currie at marc.currie@nrl.navy.mil \\ URL: https://www.jove.com/video/57103 \\ DOI: doi:10.3791/57103
}

Keywords: Engineering, Issue 135, Atomic Layer Deposition, Thin Film Growth, Low Temperature Growth, Metal-Insulator Transition, Optical Characterization, Optical Refractive Index

Date Published: 5/23/2018

Citation: Currie, M., Mastro, M.A., Wheeler, V.D. Atomic Layer Deposition of Vanadium Dioxide and a Temperature-dependent Optical Model. J. Vis. Exp. (135), e57103, doi:10.3791/57103 (2018).

\section{Abstract}

Vanadium dioxide is a material that has a reversible metal-insulator phase change near $68^{\circ} \mathrm{C}$. To grow $\mathrm{VO}_{2}$ on a wide variety of substrates, with wafer-scale uniformity and angstrom level control of thickness, the method of atomic-layer deposition was chosen. This ALD process enables high-quality, low-temperature $\left(\leq 150^{\circ} \mathrm{C}\right)$ growth of ultrathin films $(100-1000 \AA)$ of $\mathrm{VO}_{2}$. For this demonstration, the $\mathrm{VO}_{2}$ films were grown on sapphire substrates. This low temperature growth technique produces mostly amorphous $\mathrm{VO}_{2}$ films. A subsequent anneal in an ultrahigh vacuum chamber with a pressure of $7 \times 10^{-4} \mathrm{~Pa}$ of ultra-high purity $(99.999 \%)$ oxygen produced oriented, polycrystalline $\mathrm{VO}_{2}$ films. The crystallinity, phase, and strain of the $\mathrm{VO}_{2}$ were determined by Raman spectroscopy and X-ray diffraction, while the stoichiometry and impurity levels were determined by X-ray photoelectron spectroscopy, and finally the morphology was determined by atomic force microscopy. These data demonstrate the high-quality of the films grown by this technique. A model was created to fit to the data for $\mathrm{VO}_{2}$ in its metallic and insulating phases in the near infrared spectral region. The permittivity and refractive index of the ALD $\mathrm{VO}_{2}$ agreed well with the other fabrication methods in its insulating phase, but showed a difference in its metallic state. Finally, the analysis of the films' optical properties enabled the creation of a wavelength- and temperature-dependent model of the complex optical refractive index for developing $\mathrm{VO}_{2}$ as a tunable refractive index material.

\section{Video Link}

The video component of this article can be found at https://www.jove.com/video/57103/

\section{Introduction}

Vanadium dioxide undergoes a crystalline phase transition near $68^{\circ} \mathrm{C}$. This produces a structural crystal change from monoclinic to tetragonal. The origin of this transition remains controversial ${ }^{1}$, however recent research is helping develop an understanding of the processes that produce this transition ${ }^{2,3,4}$. Irrespective of the origin, the phase transition changes the optical properties of $\mathrm{VO}_{2}$ from an insulator (transmitting light) at room temperature to a more metallic material (reflecting and absorbing light) above the transition temperature ${ }^{2}$.

A variety of methods have been used to fabricate $\mathrm{VO}_{2}$ in the past (sputtering, physical vapor deposition, chemical vapor deposition, molecular beam epitaxy, solution, etc. $)^{5}$. The properties of $\mathrm{VO}_{2}$ largely depend on the technology used to fabricate the films ${ }^{6}$, which has produced significant variability between different growth techniques and subsequent anneal and led to varying crystallinity and film properties. This work investigates the optical properties of atomic layer deposited (ALD) grown films, however, the approach is applicable to modeling all types of $\mathrm{VO}_{2}$ films.

Recently, groups are constructing optical devices by incorporating thin films of $\mathrm{VO}_{2}$ onto optical substrates. As a rapidly growing new deposition method, ALD can assist in fabricating these optical devices and has several advantages over alternative techniques, such as large-area uniformity, angstrom level thickness control, and conformal film coverage ${ }^{7,8,9}$. ALD is the preferred technique for applications requiring a selflimiting layer-by-layer deposition approach, fabrication on a wide variety of substrate materials (e.g., for heterogeneous integration), or conformal coating of $3 \mathrm{D}$ structures ${ }^{10}$. Finally, the conformal coating of 3D structures of ALD process is particularly useful in optical applications.

For the experiments in this paper, ultrathin, amorphous ALD films were grown on double-side-polished, c-plane sapphire substrates at low temperatures and annealed in an oxygen environment to produce high-quality crystalline films. Using the experimental measurements, a model is created for temperature and wavelength dependent optical changes in $\mathrm{VO}_{2}$ to enable its use as a tunable refractive index material ${ }^{11}$.

\section{Protocol}

Caution: Consult all relevant material safety data sheets (MSDS) before use, and follow all appropriate safety practices and procedures. The atomic layer deposition growth of vanadium dioxide uses an ALD reactor. The precursors used for the ALD growth are tetrakis(ethylmethylamido)vanadium(IV) (TEMAV) and ozone (generated from ultra-high purity, UHP, $99.999 \%$ oxygen gas at $0.3 \mathrm{slm}$ flow and 5 psi backing pressure). In addition, UHP (99.999\%) nitrogen gas is used for purging the reactor chamber. For the subsequent vacuum 
anneal, UHP oxygen gas is used during annealing and UHP nitrogen for venting. TEMAV is flammable and should only be used with appropriate engineering controls. Compressed oxygen gas is a hazard and should only be used with appropriate engineering controls. Compressed nitrogen gas is a hazard and should only be used with appropriate engineering controls. All gases (TEMAV, oxygen, ozone and nitrogen) are connected to the ALD reactor using appropriate engineering safety controls. Stainless steel tubing connects the ozone generator to the ALD reactor, since it is cleaner and more reliable then plastic tubing. Separate UHP oxygen and nitrogen sources are connected to the vacuum annealing chamber using appropriate engineering safety controls before beginning the procedure. Acetone and 2-propanol are irritants and should only be used with appropriate personal protective equipment and safety procedures (e.g., gloves, fume hood, etc.)

\section{Atomic Layer Deposition of Vanadium Dioxide on Sapphire Substrates}

1. Clean a c-Al $\mathrm{O}_{3}$ (sapphire) substrate as follows: Solvent clean the substrate in acetone at $40{ }^{\circ} \mathrm{C}$ in sonicator for 5 min, then transfer it directly (no rinse) to 2-propanol at $40^{\circ} \mathrm{C}$ and sonicate for $5 \mathrm{~min}$. Rinse the substrate in running deionized water for 2 min, and dry it with nitrogen gas.

2. Ensure that $A L D$ reactor chamber is at $150^{\circ} \mathrm{C}$ and vent the ALD reactor with nitrogen gas.

3. Load cleaned sapphire substrate into the reactor, close the reactor, and pump to $<17 \mathrm{~Pa}$ vacuum. Wait at least $300 \mathrm{~s}$ to ensure that the sample reaches $150^{\circ} \mathrm{C}$.

4. Prepare the ALD chamber by flowing $20 \mathrm{sccm}$ UHP nitrogen into the chamber (the base pressure should not exceed $36 \mathrm{~Pa}$ ), and then pulse ozone for 15 saturated cycles, where one cycle is a 0.05 -s pulse followed by a $15 \mathrm{~s}$ purge.

5. To grow vanadium dioxide, pulse TEMAV for $0.03 \mathrm{~s}$ followed by a $30 \mathrm{~s}$ purge, then pulse ozone for $0.075 \mathrm{~s}$ followed by a $30 \mathrm{~s}$ purge. Repeat this pulse and purge cycle until desired growth is reached.

NOTE: This process is nearly linear with a growth rate of $0.7-0.9 \AA$ per cycle.

6. Remove the sample from the ALD reactor by first venting the ALD reactor chamber with UHP nitrogen gas. Place the sample on a metal plate (heat sink) to cool it to room temperature. Close the ALD reactor and pump to $<17 \mathrm{~Pa}$ vacuum. The sample now contains an amorphous vanadium oxide film on sapphire substrate.

Caution: Remove the sample carefully, since the sample is heated to $150{ }^{\circ} \mathrm{C}$.

\section{Annealing}

NOTE: $\mathrm{VO}_{2}$ films grown by the ALD technique in step 1 produce amorphous $\mathrm{VO}_{2}$. To create oriented polycrystalline $\mathrm{VO}_{2}$ films, the samples are annealed in a custom ultra-high vacuum annealing chamber with a six-way cross. To keep the annealing chamber clean, a load lock is created to insert and remove samples. A 3" diameter oxygen resistant heater is composed of a custom platinum wire heater. This heater provides radiative heating of an oxidized Inconel sled, to which the samples are mounted. The sled has high emissivity for good heat transfer from the heater to the samples.

1. Ensure that the sled is in load lock, then vent the load lock with UHP nitrogen gas, and open the load lock. Place the sample on the sled in load lock and close load lock chamber.

2. Pump the load lock to $\sim 0.1 \mathrm{~Pa}$ using roughing pump. Next, switch to turbo pump and pump load lock to $<10^{-4} \mathrm{~Pa}$. Open the gate valve and transfer the sled to the annealing chamber, and pump the annealing chamber to $<10^{-5} \mathrm{~Pa}$.

3. Flow $1.5 \mathrm{sccm}$ ultra-high purity (UHP, $99.999 \%$ ) oxygen into the annealing chamber.

NOTE: Run oxygen through $5 \mathrm{sccm}$ mass flow controller to ensure a low flow rate. The pressure must be between $1 \times 10^{-4}$ and $7 \times 10^{-4} \mathrm{~Pa}$. This pressure must be achieved before the sample reaches $150{ }^{\circ} \mathrm{C}$.

4. Heat the sled to $560^{\circ} \mathrm{C}$ (measure with pyrometer and thermocouple) using a heating ramp rate of $\sim 20^{\circ} \mathrm{C} / \mathrm{min}$. Hold the sled at $560{ }^{\circ} \mathrm{C}$ for $2 \mathrm{~h}$ (for $300 \AA$ film).

NOTE: The annealing time is thickness dependent. Empirical data suggests annealing $1 \mathrm{~h}$ for samples $<250 \AA$ thick, $2 \mathrm{~h}$ for samples $>250 \AA$ but $<500 \AA$ thick, and $3 \mathrm{~h}$ for samples $>500 \AA$ thick.

5. Quench the sample by turning off the heater and removing the sled from the heater assembly (toward load lock).

6. Keep the sample in the oxygen environment until the sample temperature becomes less than $150^{\circ} \mathrm{C}$ (e.g., use pyrometer in load lock to measure sample temperature).

NOTE: Better samples are achieved by waiting until even lower temperatures. Once the sample is $<150{ }^{\circ} \mathrm{C}$, turn off the oxygen flow and close the gate valve.

7. Vent with UHP nitrogen gas. Remove sample at $<50{ }^{\circ} \mathrm{C}$ and place the sample on metal plate (heat sink) to cool to room temperature. Close load lock with the empty sled and pump to $0.1 \mathrm{~Pa}$ using roughing pump. Switch to turbo pump and pump load lock to $<10^{-4} \mathrm{~Pa}$.

\section{Characterization}

1. Examine the sample using Raman spectroscopy with a 532-nm laser excitation source.

1. Load the sample into a microscope and bring it into focus. Verify the sample focus of the camera image in the software. Set the scan Laser Power to $4 \mathrm{~mW}$, Exposure Time to $0.125 \mathrm{~s}$, Number of Scans to 10, and preview size to $40 \mu \mathrm{m}$.

2. Click Live Spectrum to observe Raman spectrum. Optimize focus, laser power, exposure time, and number of scans to maximize signal to noise ratio. Click Save spectrum to save the data.

Open Spectrum in OMNIC. Click the "Find Pks" button to identify peaks. Determine crystallinity, phase $\left(\mathrm{VO}_{2} v_{s}\right.$. $\mathrm{VO}, \mathrm{V}_{2} \mathrm{O}_{3}, \mathrm{~V}_{2} \mathrm{O}_{5}$, etc. $)$, and strain by comparing peaks to reference data for vanadium oxides ${ }^{12,13}$.

NOTE: Narrow peaks indicate high crystalline quality, while hardening of Raman phonon modes 193,222 , and $612 \mathrm{~cm}^{-1}$ and/or softening of the $389 \mathrm{~cm}^{-1}$ mode are indicators of tensile strain in the $\mathrm{VO}_{2}$ crystal.

2. Determine orientation, crystallinity, and phase by X-ray diffraction (XRD).

NOTE: The appearance of peaks in the XRD spectra indicates the nature of the crystalline structure, specifically the crystal structure and orientation. Determine the orientation of $\mathrm{VO}_{2}$ by matching the $2 \Theta$ angle of the XRD data peak to the Inorganic Crystal Structure Database (ICSD) cards for different planes of $\mathrm{VO}_{2}$. Determine the phase by matching data peaks to cards of different vanadium oxide phases. Manual 
comparison of experimental peaks to the standard database was performed in this work. A single $\mathrm{VO}_{2}$ peak at 39.9 degrees verifies the $\mathrm{VO}_{2}$ crystal quality and demonstrates the monoclinic $(020)$ orientation.

3. Determine stoichiometry and impurity levels by X-ray photoelectron spectroscopy (XPS)

1. Load the sample onto the sample holder. Open software and click vent load lock. Insert sample holder into load lock, and click pump down. Wait until pressure $<4 \times 10^{-5} \mathrm{~Pa}$. Click the transfer button to move the sample holder into the chamber. Verify the chamber pressure is $<7 \times 10^{-6} \mathrm{~Pa}$.

2. Input measurement parameters into the experiment tree. Turn on X-ray gun with $400 \mu \mathrm{m}$ spot size and then turn on flood gun. Add a point for survey measurement, and then add points for high resolution scans of precursor elements $(\mathrm{C}$ and $\mathrm{N})$ as well as the film elements ( $\mathrm{V}$ and $\mathrm{O})$. For each measurement, add pass energy (200 eV for survey and $20 \mathrm{eV}$ for high-resolution scans) and number of scans (2 or more for survey and 15 for high resolution).

3. Place crosshairs for point measurement at the desired location on the sample. Click and highlight the experiment tree title and then click "Experimental Run" on the menu bar to execute all measurement scans.

4. Execute the survey ID procedure to identify and analyze the elements in the film. Select Manual Peak Add followed by Peak Fit buttons to analyze bonding in high-resolution data scans. Determine stoichiometry by taking the ratio of the integrated peak intensities according to the XPS Handbook ${ }^{14}$.

5. When finished, shutdown the guns, then click the transfer button to move the sample holder into the load lock. Once the sample is in the load lock, click vent load lock and unload the sample.

NOTE: This identifies the presence of all elements in survey, thereby identifying impurities. XPS peaks at specific binding energies show the valences of vanadium oxides, as shown in Figure 1. For example, these results show two representative XPS measurements obtained by depth profiling with a cluster ion gun etching the as-deposited sample. The XPS measurement of the surface shows a peak at $\sim 518 \mathrm{eV}$ demonstrating the presence of $\mathrm{V}_{2} \mathrm{O}_{5}$, while the XPS measurement of the etched sample (in the film's bulk) shows a peak at $\sim 516 \mathrm{eV}$ demonstrating the presence of $\mathrm{VO}_{2}$. The full-width-at-half-max indicates the bonding uniformity of the vanadium in the films (in other words, the phase purity). In addition, the analysis of the other energy peaks enables identification of contaminates (>1\%).

4. Determine morphology by using atomic force microscopy (AFM)

1. Turn on the computer and AFM electronics. Start the Nanoscope program and select Tapping Mode, then select load Experiment. Initialize Stage.

2. Follow the experimental order on the left side of the screen and click Setup Menu. Use focus controls to focus optics on the cantilever. Align the laser on the probe by clicking Optimize Laser Position. Click Autoalign detector button, and then click the Autotune cantilever.

3. Load sample and turn on vacuum.

4. Click Navigate button and use the trackball to place the sample under the head. Click Tip Reflection and lower the head to the sample surface using Trackball, while holding the focus button down until the tip is in focus. Click the Sample button and close the AFM hood.

5. Click the Check Parameter Menu. Ensure the scan size is $<1 \mu \mathrm{m}$ and set the samples/line to 512. Click the Engage Menu button. Wait $20 \mathrm{~s}$.

6. Set the Scan size for $3 \mu \mathrm{m}$ and leave the scan speed at $3.92 \mathrm{~Hz}$. Optimize the image, if necessary, by changing parameters: drive amplitude, amplitude set point, integral and proportional gains.

7. Capture the desired image by clicking the Frame Down button followed by the Capture Button. Following the scan, click the Withdraw button.

8. Double click on the desired image to open it in analysis software. To determine morphology, click the Flatten button and then click execute. Extract statistical parameters by clicking Roughness to calculate surface roughness and click Particle Analysis to calculate the depth histogram and mean grain size.

9. Click the Navigate Menu and click the Sample Load Position. Turn off the vacuum and unload the sample.

NOTE: For consistency from sample to sample, keep the same AFM scan parameters.

5. Determine optical transmittance and reflectance.

1. Use a tunable optical source and measure optical transmittance and reflectance at (or near) normal incidence in the near-infrared region. Calibrate the system without sample for $100 \%$ transmittance and with a gold mirror for near $100 \%$ reflectance.

2. Load the $\mathrm{VO}_{2}$ sample onto the temperature controlled stage. Stabilize the sample at the desired temperature. Measure transmittance and reflectance over the desired spectral range, for example, throughout the near infrared region.

NOTE: For optimal results, the temperature should be varied at least $20^{\circ} \mathrm{C}$ above and below the transition temperature of $\mathrm{VO}_{2}$ (usually, $\left.68^{\circ} \mathrm{C}\right)$.

\section{Modelling Optical Constants (Permittivity and Refractive Index)}

1. Model the complex dielectric permittivity, $\varepsilon$, as a function of photon energy, $E$, using the following equation (where $\varepsilon_{\infty}$ is the high frequency permittivity followed by the sum of $n$ oscillators where $A_{n}$ is the oscillator amplitude, $E_{n}$ the oscillator energy, and $B_{n}$ the oscillator damping):

$\varepsilon\{E\}=\varepsilon_{\infty}+\sum_{n} \frac{A_{n}}{\left(E_{n}^{2}-E^{2}\right)-i E B_{n}}$

NOTE: Custom Matlab programs analyzed and modelled the data.

2. Enter known parameters for sapphire substrate in step 4.1, and use these to calculate the dielectric permittivity as a function of optical wavelength. Then calculate the refractive index $(n=\sqrt{ } \varepsilon)$, and using the refractive index, calculate the reflectance and transmittance of the substrate.

3. Compare the results of step 4.2 with the measured data, and then employ an optimization technique (such as Nelder Mead ${ }^{15}$ ) to update the input parameters of step 4.2 in order to reduce the error between the measured and calculated results, thereby optimizing the parameters for the sapphire substrate.

4. Estimate parameters for $\mathrm{VO}_{2}$ in the equation in step 4.1, and use these to calculate the dielectric permittivity. Then calculate the refractive index $(n=\sqrt{\varepsilon})$ and use that, along with the $\mathrm{VO}_{2}$ thickness, substrate thickness, refractive index (from step 4.3), optical wavelength, optical

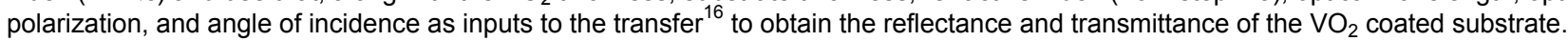


Employ an optimization technique (such as Nelder $\mathrm{Mead}^{15}$ ) to update the $\mathrm{VO}_{2}$ input parameters and reduce the error between the measured and calculated results, thereby optimizing the parameters for the $\mathrm{VO}_{2}$.

5. Perform steps 4.2 to 4.4 at several temperatures spanning the insulating and metallic states of vanadium dioxide (temperatures from 30 to 90 $\left.{ }^{\circ} \mathrm{C}\right)$. Model the temperature dependence of the refractive index of $\mathrm{VO}_{2}$ as follows:

$\varepsilon_{V O 2}(T)=f(T) \times \varepsilon_{\text {ins }}+(1-f(T)) \times \varepsilon_{\text {metal }}$

where $\varepsilon_{V O 2}(T)$ is the refractive index of $\mathrm{VO}_{2}$ as a function of temperature, $\varepsilon_{\text {ins }}$ and $\varepsilon_{\text {metal }}$ are the dielectric permittivity of the insulating and metallic phases, and $f(T)$ is a temperature-dependent function governing the distribution of insulating and metallic optical properties. Use a Fermi-Dirac-like function for $f(T)$ to govern the transition between insulating and metallic state, similar to effective-medium approximations $^{17,18,19}$, given by:

$f(T)=1 /\left(\exp \left(\frac{T-T_{t}}{W \cdot T_{t}}\right)+1\right)$

where $T_{t}$ is the transition temperature and $W$ controls the width of the transition.

6. Employ an optimization technique to update the parameters (W and $T \mathrm{t}$ ) in the equation for $f(T)$ to reduce the error between the measured and calculated results, thereby optimizing the transition temperature and width. This results in a temperature and wavelength dependent model for the dielectric permittivity and refractive index of $\mathrm{VO}_{2}$.

\section{Representative Results}

To identify the quality of the ALD grown vanadium oxide, X-ray photoelectron spectroscopy (XPS) was performed on the as-deposited, primarily amorphous $\mathrm{VO}_{2}$ films (Figure 1) as well as annealed crystalline $\mathrm{VO}_{2}$ films (not shown). X-ray diffraction (XRD) was performed on the annealed $\mathrm{VO}_{2}$ films (Figure 2). In addition, to help quantify the vertical profile of the chemistry within the film, depth profiling was performed with a cluster ion source to minimize preferential etching of cation/anion species. Two representative traces are shown in Figure 1, one at the surface and one in the bulk. The depth profile and subsequent XPS measurements show that the top 1-nm of the as-deposited film is not $\mathrm{VO}_{2}$ due to excess environmental (adventitious) oxygen and carbon, but after a more controlled annealing procedure in low-pressure oxygen even the surface stabilizes to $\mathrm{VO}_{2}$. X-ray diffraction measurements were performed with a Cu K-alpha X-ray energy source and show, in Figure 2, a single $\mathrm{VO}_{2}$ peak at $39.9^{\circ}$. The signature of this peak verifies the quality of the ALD-grown $\mathrm{VO}_{2}$ as well as that the (020) crystal orientation aligns with the sapphire substrate's peak.

To analyze the crystallinity, phase, and strain, Raman spectroscopy was performed using a 532-nm laser for excitation. Figure 3 shows a Raman spectrum of the $\mathrm{VO}_{2}$ film and shows narrow peaks which indicate high crystalline quality. In addition, the increased energy in the vanadiumvanadium low-frequency phonons $\left(193\right.$ and $\left.222 \mathrm{~cm}^{-1}\right)$ and the $612 \mathrm{~cm}^{-1}$ mode, as well as the decreased energy of the $389 \mathrm{~cm}^{-1}$ mode, suggest tensile strain in these films ${ }^{12,13}$.

The morphology was observed by atomic force microscopy (AFM). Figure 4 shows crystal grain sizes on the order of 20-40 $\mathrm{nm}$ and a rootmean-square (RMS) roughness of $1.4 \mathrm{~nm}$ for as-deposited films (Figure 4A) and an RMS roughness of $2.6 \mathrm{~nm}$ for annealed films (Figure 4B).

Optical transmittance and reflectance data were obtained using a white light source with a scanning monochromator and a photodetector, which provided coverage in the visible and near infrared region. Figure 5 shows the temperature dependence of the film as it transitions from an insulator to a metal, demonstrating a transition temperature of $61^{\circ} \mathrm{C}$. Analyzing the experimental data enables the modeling of the temperature and wavelength dependent permittivity of the $\mathrm{VO}_{2}$ as it transitions from insulator to metal. Figure $\mathbf{5}$ shows how the model accurately predicts the optical behavior when using the parameters in Table 1. 


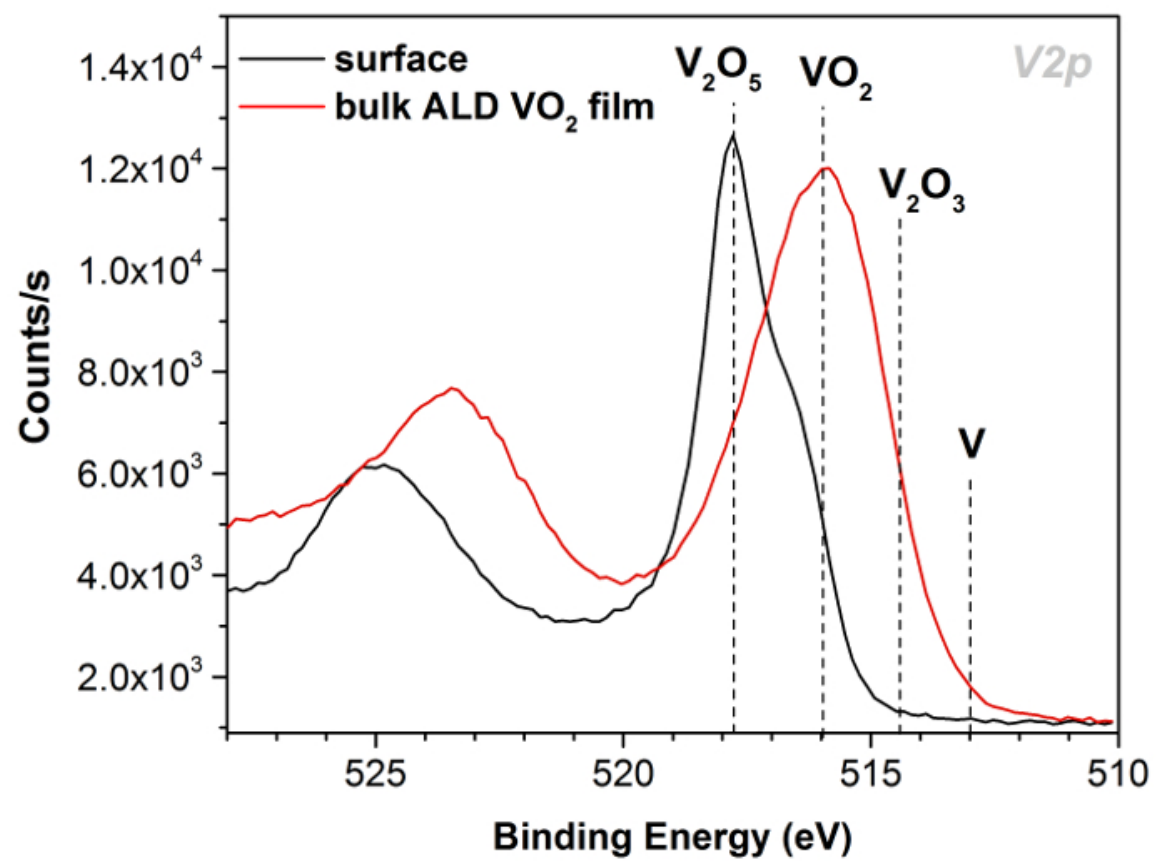

Figure 1: Representative XPS measurements of 35-nm thick $\mathrm{VO}_{2}$ on $\mathrm{C}-\mathrm{Al}_{2} \mathrm{O}_{3}$. XPS shows that the bulk of the film is $\mathrm{VO}_{2}$ while the surface, which contains $\mathrm{C}$ and $\mathrm{O}$ contaminates, is shifted more towards $\mathrm{V}_{2} \mathrm{O}_{5}$. Stoichiometry suggests $\mathrm{VO}_{2}$. Please click here to view a larger version of this figure.

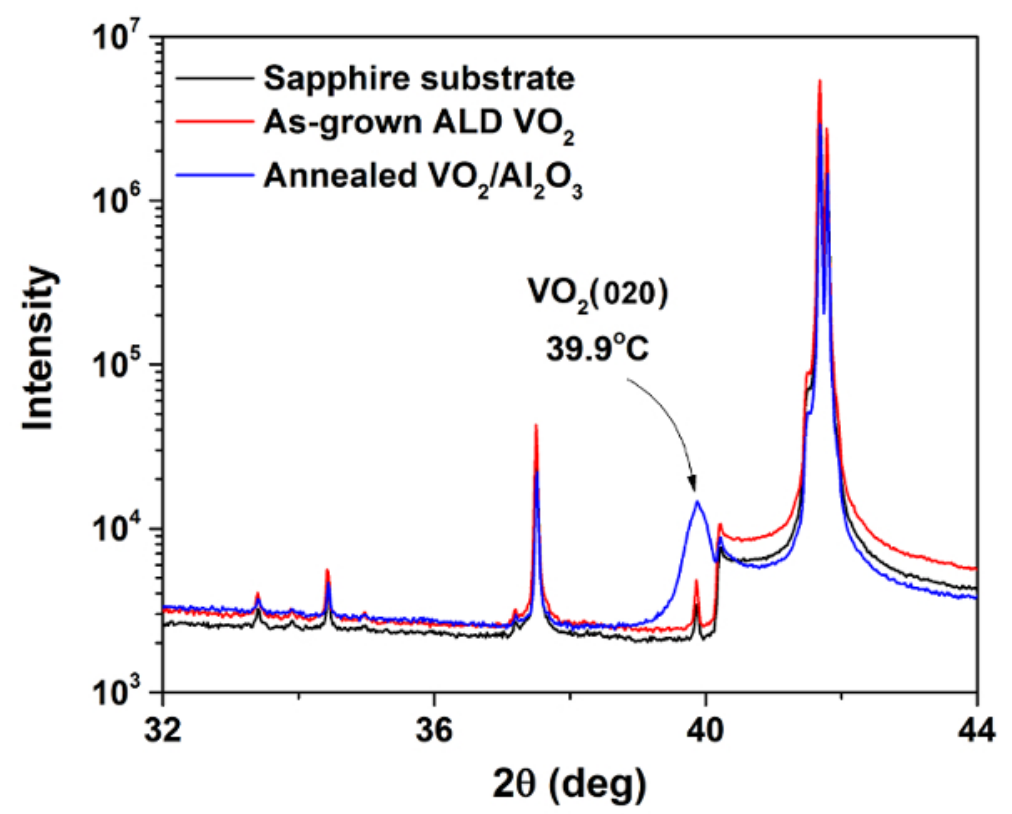

Figure 2: XRD measurements of $35-\mathrm{nm}$ thick $\mathrm{VO}_{2}$ on $\mathrm{c}-\mathrm{Al}_{2} \mathrm{O}_{3}$. This $\mathrm{XRD}$ measurement shows a single $\mathrm{VO}_{2}$ peak at $39.9^{\circ}$ that independently verifies the crystal quality and demonstrates the monoclinic (020) orientation is aligned with the underlying sapphire peak. Please click here to view a larger version of this figure. 


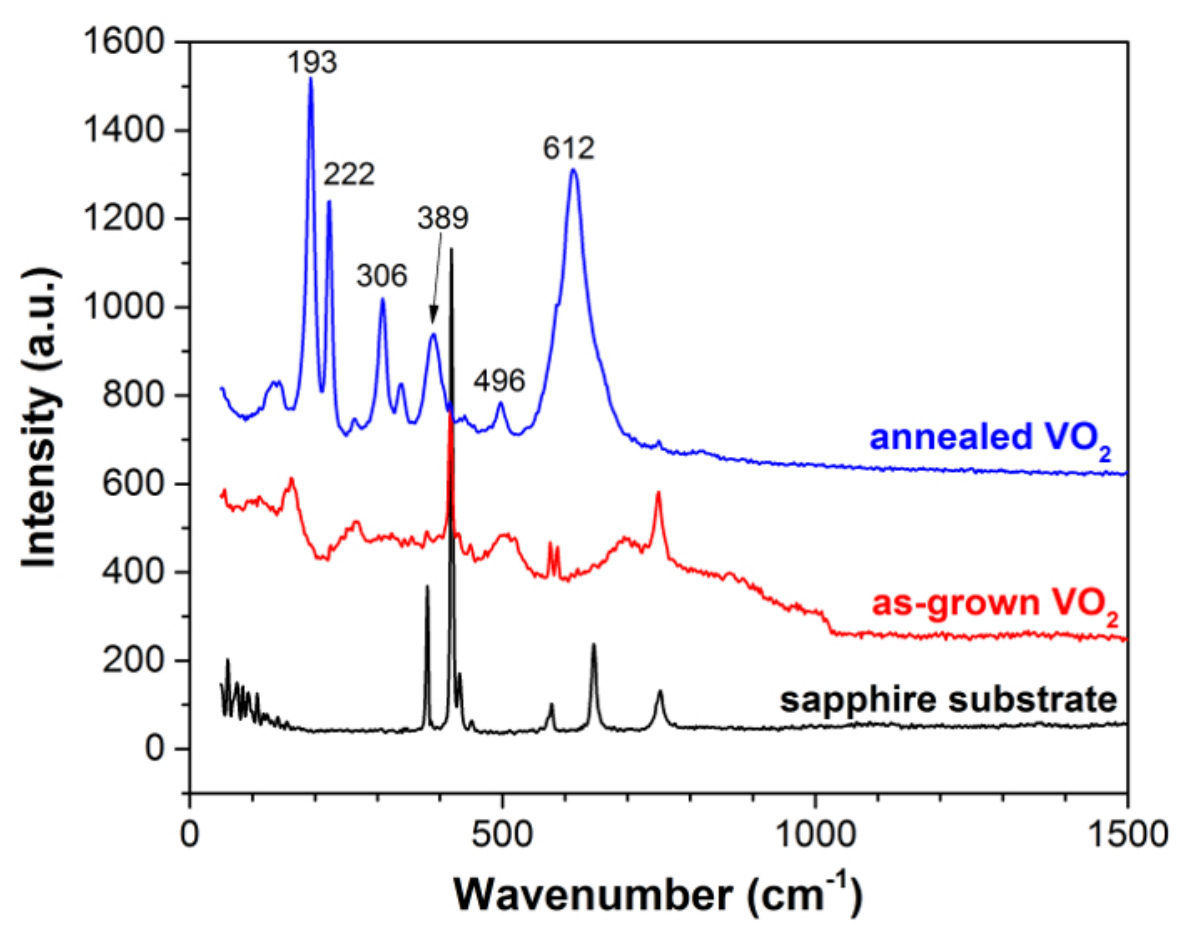

Figure 3: Raman spectra of $\mathrm{VO}_{2}$ on $\mathrm{c}-\mathrm{Al}_{2} \mathrm{O}_{3}$. This Raman spectrum has narrow peaks, indicating high crystalline quality, and shows a slight tensile strain. Please click here to view a larger version of this figure.
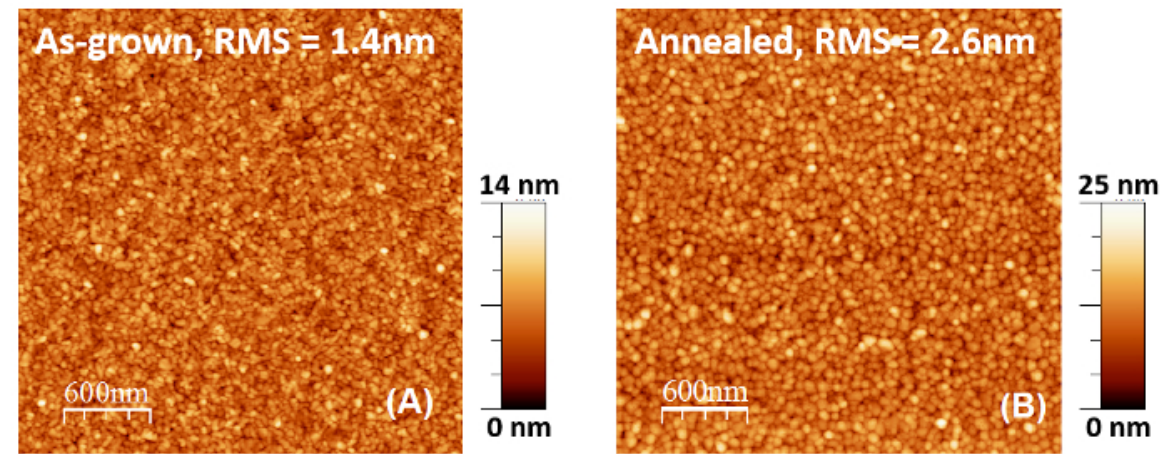

Figure 4: Morphology of $\mathrm{VO}_{2}$ on $\mathrm{C}-\mathrm{Al}_{2} \mathrm{O}_{3}$. The AFM images show uniform, continuous films with grain sizes on the order of $20-40 \mathrm{~nm}$ and $\mathrm{RMS}$ roughnesses of $(\mathbf{A}) 1.4 \mathrm{~nm}$ for the as-grown film and (B) $2.6 \mathrm{~nm}$ for the annealed film. Please click here to view a larger version of this figure. 

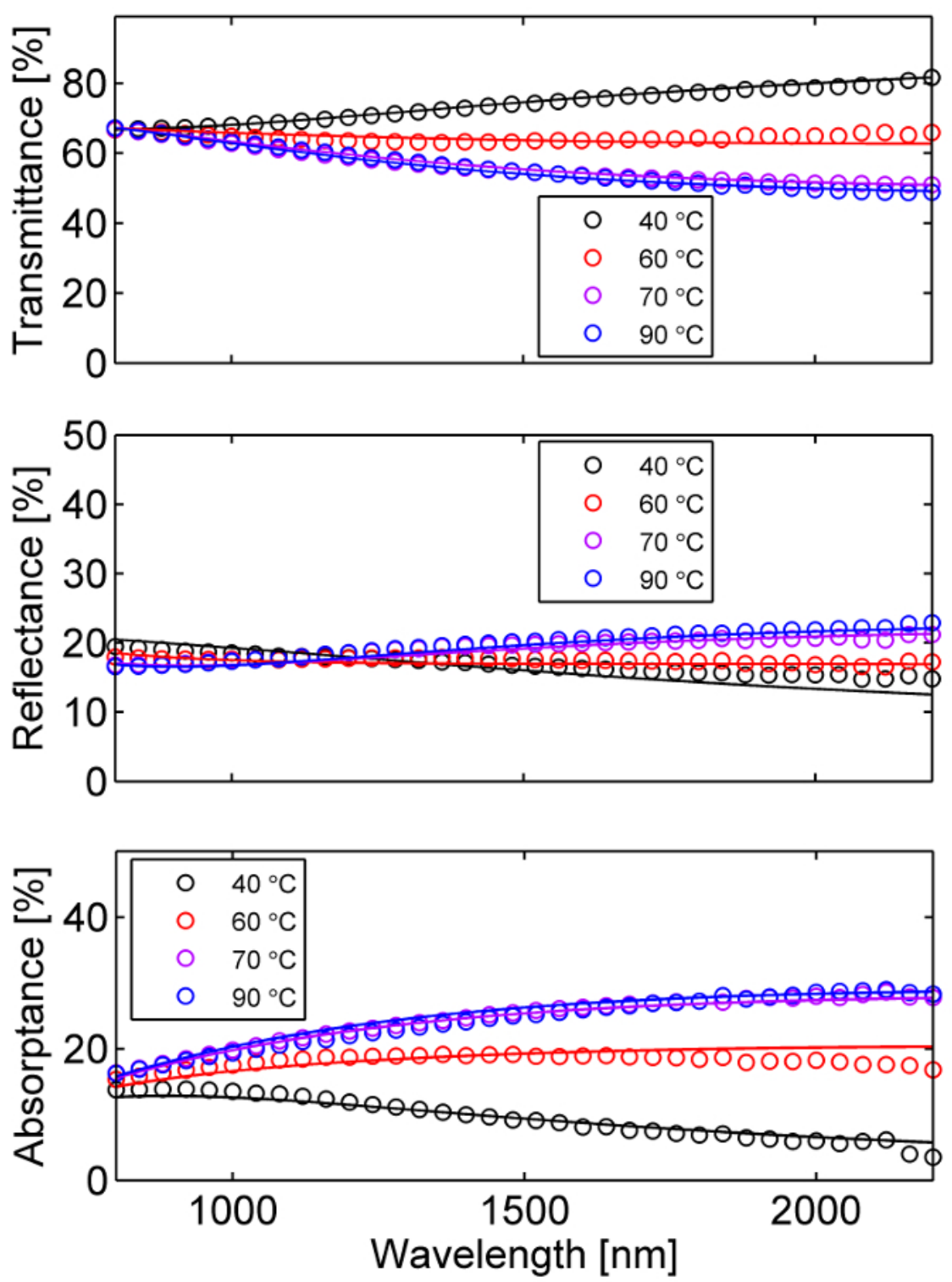

Figure 5: Near-infrared optical transmittance and reflectance of 35-nm thick $\mathrm{VO}_{2}$ on $\mathrm{c}_{-} \mathrm{Al}_{2} \mathrm{O}_{3}$. The temperature-dependent behavior of the optical transmittance and reflectance of vanadium dioxide film are show at $40,60,70$, and $90^{\circ} \mathrm{C}$. The open circles in the plot are the measured transmittance, reflectance, and calculated absorptance of the $\mathrm{VO}_{2}$ on sapphire structure at various temperatures, while the solid lines are the predicted values from the two-dimensional temperature- and wavelength-dependent model of $\mathrm{VO}_{2}$. Please click here to view a larger version of this figure. 


\begin{tabular}{|c|c|c|c|c|}
\hline & $\varepsilon_{\infty}$ & & osc. 1 & osc. 2 \\
\hline \multicolumn{5}{|l|}{ Insulator } \\
\hline & \multirow[t]{3}{*}{3.4} & $E_{n}$ & 3.8 & 1.2 \\
\hline & & $A_{n}$ & 33 & 2.1 \\
\hline & & $B_{n}$ & 1.4 & 1.3 \\
\hline \multicolumn{5}{|l|}{ Metal } \\
\hline & \multirow[t]{3}{*}{4.5} & $E_{n}$ & 3.2 & 0.6 \\
\hline & & $A_{n}$ & 13 & 5.3 \\
\hline & & $\mathrm{B}_{\mathrm{n}}$ & 1.1 & 1 \\
\hline
\end{tabular}

Table 1: Representative model parameters for $\mathrm{VO}_{2}$. These parameters are representative of those used in an oscillator model to estimate the permittivity of $\mathrm{VO}_{2}$ in its metallic and insulating phases.

\section{Discussion}

The growth methods described here provide reproducible results with regards to uniformity, chemistry, structure, and morphology. The vanadium precursor is critical to producing the correct stoichiometry of as-deposited ALD films. This particular precursor promotes the +4 vanadium valence state, unlike many of the others listed in the literature that promote the more common +5 valence state. Additionally, this particular precursor has a fairly low vapor pressure and requires heating to provide a sufficient dose to saturate under the conditions given. Since this precursor starts to degrade around $175^{\circ} \mathrm{C}$, this sets an upper temperature limit to both heating of the precursor and ALD growth. Another critical aspect to achieving correct stoichiometry is the ozone concentration $(\sim 125 \mathrm{mg} / \mathrm{L}$ here $)$ during dosing. Often the concentration of ozone produced by a generator under particular conditions degrades or drifts over time. If this happens, the ozone pulse and purge durations will have to be adjusted to maintain stoichiometry, morphology, and wafer uniformity. What is described here is how to grow ALD VO ${ }_{2}$ on c-plane sapphire substrates, which includes in-situ ozone pre-treatment. The steps prior to growth for cleaning and nucleation are dependent on the substrate; however, the process described here works for most substrates (inert, oxides, metals, etc.) To determine the best termination cleaning and preparation for $\mathrm{VO}_{2}$ growth, one should consider reactivity between species termination and the vanadium precursor while minimizing any native oxide on the substrate. Finally, this process has been demonstrated on high aspect ratio substrates (up to $~ 100$ ) but for extreme cases, one should consider an exposure or static ALD method to enhance conformality further.

The ability to achieve high quality, crystalline ALD VO $\mathrm{O}_{2}$ films is quite dependent on the post-deposition annealing parameters. The most critical aspect is the pressure, specifically the partial pressure of oxygen. High oxygen pressures lead to faceting and grain growth, eventually causing nanowire formation, as well as results in the $\mathrm{V}_{2} \mathrm{O}_{5}$ phase. If the oxygen pressure is too low, oxygen is annealed out of the films resulting in $\mathrm{V}_{2} \mathrm{O}_{3}$ phase. Thus, to maintain the correct phase and minimize film roughness, the oxygen pressure should be maintained in the range of $1 \times 10^{-4}$ to $7 \times 10^{-4} \mathrm{~Pa}$. Similarly, the temperature is critical to both being able to crystallize the film, maintain stoichiometry, and minimize roughening of the film. While the temperature of the $\mathrm{VO}_{2}$ film is difficult to measure, empirical findings suggest that crystallization requires stage temperatures greater than $500^{\circ} \mathrm{C}$. At higher temperatures, it is harder to maintain the correct stoichiometry and phase and produce pinhole free films. There is also a trade-off between temperature and anneal time, specifically higher temperatures can reduce the anneal time. Additionally, the anneal duration is directly tied to the thickness of the film. Thicker films require longer times to achieve maximum crystallization. Thus, the oxygen pressure, anneal temperature, and anneal time described in the methods above were optimized to produce high quality $\mathrm{VO}_{2}$ films that exhibit the largest change in optical properties at a nearly ideal transition temperature. Finally, the ramping and cooling rates during the oxygen anneal have an effect on roughness and morphology; the slower these are, the smoother the films.

ALD deposition and subsequent anneal of $\mathrm{VO}_{2}$ produces oriented polycrystalline films with large area uniformity. ALD offers conformally grown films on three-dimensional nanoscale morphologies of almost any substrate. This enables $\mathrm{VO}_{2}$ integration into novel applications, and is especially well suited for optical devices.

Following growth and optical measurements, a model is created which provides a good fit to the data for both the transmittance and reflectance of $\mathrm{VO}_{2}$ in its metallic and insulating phases in the near infrared spectral region $\left(\mathrm{R}^{2}=0.96-0.99\right)$. The reflectance of the infrared insulating phase is the most challenging process in creating this model. Additional oscillator terms were added, but this increased model complexity, only marginally improve the fit in this region. It should be noted that in this model, the superposition of Lorentz oscillators is a common optical model and do not necessarily correspond to specific electronic transitions. Initially, the models included a Drude term, however, after mathematical optimization, the Drude term was essentially eliminated. For this reason, several minimization techniques were examined. However, these different techniques converged upon similar solutions that did not involve a Drude term. The absence of a Drude term in the ALD VO ${ }_{2}$ could be due to a number of factors, such as 1) doped-semiconductor-like resistivity, or 2) a plasma frequency shift to lower energies and/or large collision rate (damping term), in agreement with the metallic properties of these films.

In the insulating phase, $\mathrm{T}<60^{\circ} \mathrm{C}$, the permittivity and refractive index of the $\mathrm{ALD} \mathrm{VO}$ agree well with the other fabrication methods (sputtered $^{4,20,21}$ and pulsed-laser deposition ${ }^{22,23}$ ). In the metallic state, $\mathrm{T}>70^{\circ} \mathrm{C}$, these ALD films exhibit lower loss than the $\mathrm{VO}_{2}$ fabricated by other methods. It is important to note that while different fabrication methods produce somewhat different values for the permittivity and refractive index of $\mathrm{VO}_{2}$, all films show similar trends.

The model in this paper of the temperature and wavelength dependence of the optical permittivity and refractive index agrees well with the experimentally measured data. This model's ability to produce a good quality fit to the measured optical data demonstrates it can reliably predict the optical properties of $\mathrm{VO}_{2}$ as the phase changes from an insulator to a metal. Using these models, the optical properties of $\mathrm{VO}_{2}$ can be predictably tuned by temperature, thickness, and wavelength to design optical systems that achieve static and dynamic goals. These models 
enable the design and development of optical systems using $\mathrm{VO}_{2}$ in passive and active systems by modifying the film's thickness as well as temperature.

\section{Disclosures}

The authors have nothing to disclose.

\section{Acknowledgements}

This work was supported by core programs at the U.S. Naval Research Laboratory.

\section{References}

1. Park, J.H. et al. Measurement of a solid-state triple point at the metal-insulator transition in VO2. Nature. 500 (7463), $431-434$ (2013)

2. Yang, Z., Ko, C., Ramanathan, S. Oxide Electronics Utilizing Ultrafast Metal-Insulator Transitions. Annu. Rev. Mater. Res. 41 (1), $337-367$ (2011).

3. Tan, X. et al. Unraveling Metal-insulator Transition Mechanism of $\mathrm{VO}_{2}$ Triggered by Tungsten Doping. Sci. Rep. 2, srep00466 (2012).

4. Verleur, H.W., Barker, A.S., Berglund, C.N. Optical Properties of $\mathrm{VO}_{2}$ between 0.25 and 5 eV. Phys. Rev. 172 (3), $788-798$ (1968).

5. Kiria, P., Hyett, G., Binions, R. Solid State Thermochromic Materials. Adv. Mater. Lett. 1 (2), $86-105$ (2010).

6. Konovalova, O.P., Sidorov, A.I., Shaganov, I.I. Interference systems of controllable mirrors based on vanadium dioxide for the spectral range 06-106 micrometer. J. Opt. Technol. 66 (5), 391 (1999).

7. Premkumar, P.A. et al. Process Study and Characterization of $\mathrm{VO}_{2}$ Thin Films Synthesized by ALD Using TEMAV and $\mathrm{O}_{3}$ Precursors. ECS $\mathrm{J}$. Solid State Sci. Technol. 1 (4), P169-P174 (2012).

8. Rampelberg, G. et al. Crystallization and semiconductor-metal switching behavior of thin $\mathrm{VO}_{2}$ layers grown by atomic layer deposition. Thin Solid Films. 550, 59-64 (2014).

9. Peter, A.P. et al. Metal-Insulator Transition in ALD VO 2 Ultrathin Films and Nanoparticles: Morphological Control. Adv. Funct. Mater. 25 (5), 679-686 (2015).

10. Rampelberg, G. Thin film synthesis of $\mathrm{VO}_{2}$ and $\mathrm{VN}$ by gas-solid reactions and atomic layer deposition. (2016).

11. Kats, M.A. et al. Vanadium Dioxide as a Natural Disordered Metamaterial: Perfect Thermal Emission and Large Broadband Negative Differential Thermal Emittance. Phys. Rev. X. 3 (4), 041004 (2013).

12. Atkin, J.M. et al. Strain and temperature dependence of the insulating phases of $\mathrm{VO}_{2}$ near the metal-insulator transition. Phys. Rev. B. 85 (2), 020101 (2012).

13. Petrov, G.I., Yakovlev, V.V., Squier, J. Raman microscopy analysis of phase transformation mechanisms in vanadium dioxide. Appl. Phys. Lett. 81 (6), 1023-1025 (2002).

14. Moulder, J.F. Handbook of X-ray Photoelectron Spectroscopy: A Reference Book of Standard Spectra for Identification and Interpretation of XPS Data. Physical Electronics Division, Perkin-Elmer Corporation. (1992).

15. Nelder, J.A., Mead, R. A Simplex Method for Function Minimization. Comput. J. 7 (4), 308-313 (1965).

16. Born, M., Wolf, E. Principles of Optics: Electromagnetic Theory of Propagation, Interference and Diffraction of Light. Cambridge University Press. (1999).

17. Choi, H.S., Ahn, J.S., Jung, J.H., Noh, T.W., Kim, D.H. Mid-infrared properties of a $\mathrm{VO}_{2}$ film near the metal-insulator transition. Phys. Rev. B. 54 (7), 4621-4628 (1996)

18. Jepsen, P.U. et al. Metal-insulator phase transition in a $\mathrm{VO}_{2}$ thin film observed with terahertz spectroscopy. Phys. Rev. B. 74 (20), 205103 (2006).

19. Rozen, J., Lopez, R., Haglund, R.F., Feldman, L.C. Two-dimensional current percolation in nanocrystalline vanadiumdioxide films. Appl. Phys. Lett. 88 (8), 081902 (2006).

20. Tazawa, M., Jin, P., Tanemura, S. Optical constants of $\mathrm{V}_{1-\mathrm{x}} \mathrm{W}_{\mathrm{x}} \mathrm{O}_{2}$ films. Appl. Opt. 37 (10), 1858-1861 (1998).

21. Joushaghani, A. Micro- and Nano-scale Optoelectronic Devices Using Vanadium Dioxide. at <https://tspace.library.utoronto.ca/ handle/1807/68313> (2014).

22. Briggs, R.M., Pryce, I.M., Atwater, H.A. Compact silicon photonic waveguide modulator based on the vanadium dioxide metal-insulator phase transition. Opt. Express. 18 (11), 11192-11201 (2010).

23. Dicken, M.J. et al. Frequency tunable near-infrared metamaterials based on $\mathrm{VO}_{2}$ phase transition. Opt. Express. 17 (20), 18330 (2009). 